Veritas E Scientia

Vol. 9. $\mathrm{N}^{\circ} 2$

Julio - Diciembre del 2020

ISSN Edición Online: 2617-0639

https://doi.org/10.47796/ves.v9i2.394

Artículo original

\title{
ANÁLISIS DE ZONAS GEOGRÁFICAS PARA EL DESARROLLO DE LAS ENERGÍAS RENOVABLES NO CONVENCIONALES, EN LA REGIÓN DE ARICA Y PARINACOTA, CHILE 2019.
}

\author{
ANALYSIS OF GEOGRAPHICAL AREAS FOR THE DEVELOPMENT OF NON- \\ CONVENTIONAL RENEWABLE ENERGIES, IN THE REGION OF ARICA AND \\ PARINACOTA, CHILE 2019.
}

Claudio Marcelo Martínez Villalobos ${ }^{1}$
Angel Canales Gutiérrez ${ }^{2}$

Aceptado: 03/07/2020

Publicado online: $14 / 12 / 2020$

\begin{abstract}
RESUMEN
La investigación se realizó con el objeto de analizar la existencia de zonas geográficamente inmejorables en la región para el desarrollo de energías renovables no convencionales (ERNC), para ello se monitorearon diferentes puntos en la región de XV de Arica y Parinacota de Chile, como pampa Dos Cruces, pampa Camarones y cuesta El Águila, en un periodo de 9 meses, para consolidar esta investigación se construyeron tablas comparativas de acuerdo a los objetivos específicos correlacionando los factores climáticos de acuerdo a las horas sol versus radiación solar, vinculados a las diferentes zonas geomorfológicas de la región. Se tomaron en cuenta datos históricos de la Dirección General de Aeronáutica Civil (DGAC), los que fueron comparados y procesados mediante metodología correlacional con los datos obtenidos de los puntos meteorológicas de las zonas de interés. Luego de haber realizado el procesamiento de datos se puede concluir que existe una directa relación de los elementos meteorológicos con la geomorfología del lugar, donde se pueden implementar proyectos de Energías Renovables No Convencionales (ERNC).
\end{abstract}

Palabras claves: Irradiancia solar, Geomorfología, Factores Climáticos.

\begin{abstract}
This research was carried out in order to analyze the existence of geographically unbeatable areas in the region for the development of non-conventional renewable energies (ERNC), for this purpose different points were monitored in the XV region of Arica and Parinacota of Chile, as Pampas Dos Cruces, Pampas Camarones and El Águila costs, in a period of 9 months, to consolidate this research comparative tables were constructed according to the specific objectives correlating the climatic factors according to the sun hours versus solar radiation, linked to the different geomorphological zones of the region. Historical data from the General Directorate of Civil Aeronautics (DGAC) were taken into account, which were compared and processed using a correlational methodology with the data obtained from the meteorological
\end{abstract}

\footnotetext{
1Arquitecto de la Universidad del Mar y Magíster en Gestión Ambiental y Desarrollo Sostenible. claudio2428@yahoo.es (iD) 0000-0002-3096-1705

2 Licenciado en Biología, Magíster en Manejo de Vida Silvestre y Doctor en Ciencias y Tecnologías Medio

Ambientales. acanales7@hotmail.com (D) 0000-0003-3784-1199
} 
points of the areas of interest. After having performed the data processing it can be concluded that there is a direct relationship of the meteorological elements with the geomorphology of the place, where projects of Non-Conventional Renewable Energies (NCRE) can be implemented.

Keywords: Solar irradiance, Geomorphology, Climate Factors.

\section{INTRODUCCIÓN}

Se realiza el análisis comparativo de la oferta Regional de áreas concesionadas por parte del Ministerio de Bienes Nacionales de Chile, para Instalar Proyectos de Energía Renovable No Convencionales (ERNC), del tipo fotovoltaico. Para esto existen varios sistemas de información geográfica (SIG), que pueden ser utilizados para los análisis de potenciales de las fuentes renovables de energía. El ordenamiento territorial como disciplina científica, técnica, administrativa y política, posee un enfoque multidisciplinario, permitiendo ordenar el territorio para lograr un desarrollo social y económico equilibrado de las regiones, el mejoramiento de la calidad de vida, la gestión responsable de los recursos naturales y la protección del medio ambiente. A nivel local, Arica y Parinacota es una de las regiones que tiene uno de los suministros eléctricos más precarios, existiendo poca capacidad de generación y la conexión básicamente por la conductividad y las pérdidas. Estas últimas dependen mucho de la distancia y en grado muy importante de la temperatura, en días calurosos las pérdidas de transmisión y transformación se disparan. La región de Arica y Parinacota, en especial la ciudad de Arica, recibe casi el $80 \%$ de su suministro normal por una línea de 220 kv y otra de 110 kv, ambas de la generadora Central Térmica de Tarapacá a más de $300 \mathrm{Km}$ de distancia. Dicha central además debe alimentar tanto a las mineras de la Primera Región, como a la ciudad de Iquique. Si bien, la región posee dos pequeñas generadoras que sirven como apoyo, un diésel en Arica y una pequeña hidroeléctrica de paso en la localidad de Chapiquiña, entre ambas cubren apenas el $50 \%$ del consumo de la región. Dadas las condiciones climáticas se posee un gran potencial para la producción de electricidad, calor y luz sobre la base de fuentes de recursos energéticos como la energía solar y en menor medida eólica. A esto se agrega que más del $60 \%$ de los suelos de Arica y Parinacota son fiscales, por lo cual existe el espacio necesario para desarrollar plantas de energía. Se puede comprobar que el desierto de Atacama se encuentra en el primer lugar de radiación con un área menor de superficie requerida para generar una unidad de energía. Particularmente, en los estudios realizados por el Departamento de Geofísica de la Universidad de Chile entre los años 2009 y 2010, se encontró que la máxima radiación global horizontal de cielo despejado se encuentra en la zona cordillerana del Norte de Chile y presenta valores de radiación entre los más grandes del planeta.

En Arica norte de Chile, podemos mencionar, un aumento promedio en las dosis eritémicas de un $5.6 \% / \mathrm{km}$, y que en el modelo teórico predice valores superiores a 20 (en una escala dónde valores de índice ultravioleta superiores a 14 son considerados extremos (Rivas et al, 2002 pp. 59-62). En Chile, se desarrolló una investigación experimental a mediodía solar en Arica, norte de Chile, obtenidas por conversión de las mediciones de irradiancia solar UVB de un instrumento Solar Light 501. Se observan niveles altos y muy altos de ISUV durante gran parte del año. En particular, de los nueve meses de los cuales se presentan datos, se observan registros superiores o iguales a ISUV = 7 (Alto) durante de 7 meses, también durante tres meses se observan un valor medio de ISUV mayor que 9.9, lo cual corresponde a una calificación Muy Alto (Rivas A. et al, 2002 p.61). Sarricolea ejecutó un análisis de las tendencias de la precipitación en el norte grande de Chile y su relación con las proyecciones de cambio climático. Se comparó cómo las tendencias en la precipitación se asemejan a alguno de los más de treinta modelos proyectados de cambio climático (Sarricolea, et al, 2017 pp.41-50). 
Pizarro, encontró que el nivel del mar no se relaciona bien con el viento, aunque dentro de ciertas bandas de frecuencia se observaron valores significativos. En Iquique y Antofagasta destaca la banda centrada a los $0,13 \mathrm{cpd}(7,7$ días) con valores de coherencia significativos a un 99\% de confianza. Esta relación evidenciaría la existencia de un forzamiento local sobre las variaciones del nivel del mar (Pizarro, et al, 1994 pp.85-101). Aguilar muestra que los factores tectónicos, serían el control de primer orden en la segmentación geomorfológica del antearco, mientras que el control climático en las variaciones de la geomorfología se supedita al modelado glacial de valles que en respuesta al estímulo tectónico se encumbran sobre la mínima altitud de la línea de equilibrio glacial (Aguilar, et al, 2013). Sarricolea \& Romero concluyó que, si bien la precipitación del Altiplano del norte de Chile se concentra en verano, es posible encontrar rachas de días secos de más de un mes en dicho período (Chucuyo Retén, Colchane, Cancosa y Coyacagua), los cuales según estos resultados se pueden incrementar en cerca de 18 días en el peor escenario de cambio climático, y en el período 2061-2080. Ello tendría graves repercusiones en los ecosistemas de humedales y salares y obviamente, en las actividades económicas de ganadería y minería (Sarricolea \& Romero, 2015 pp.169-183).

En Perú Camayo, et al (2015), realizaron un estudio denominado Desarrollo del modelo bristow campbell para estimar la radiación solar global de la región de junin, Perú, que entrego como referencia que la cuantificación de la disponibilidad de la energía solar sirve de base para que sea posible el diseño de políticas y medidas para incentivar el mayor uso de estas energías limpias que promuevan el desarrollo sostenible de la Región Junín y de todo Perú (Lapa, et al, 2019 pp. 220-234). En Argentina, se desarrolló una investigación sobre radiación solar horaria, donde los resultados obtenidos muestran que sin datos de heliofanía también es posible estimar la radiación solar horaria satisfactoriamente a partir de variables normalmente provistas por estaciones meteorológicas de uso extendido (Sayago, et al 2011). En Colombia, se desarrolló un estudio sobre la Evaluación de un modelo estadístico para estimar la radiación solar en Magdalena, Colombia, el cual concluyó que cuanto a las correlaciones estadísticas, se puede concluir que los datos recopilados y observados no presentan un ajuste significativamente estadístico en los diferentes tipos de modelos estudiados. Sin embargo, sí se puede establecer que la humedad relativa guarda una mayor relación o ajuste en los modelos con la radiación solar permitiendo concluir que es la variable climatológica que más influencia tiene sobre el modelo (Velez et al, 2015 pp. 54-63). En Argentina, se desarrolló un trabajo denominado Estudio Potencial de Radiación Solar y de la Temperatura Ambiente en Lorica-Córdoba, donde sus resultados indicaron que se encontró que durante el pasado año la radiación solar diaria que en promedio incidió en la región de Lorica fue de $4898 \mathrm{w} / \mathrm{m} 2$. Este resultado muestra que el potencial de radiación solar de esta región es significativamente alto, lo cual indica que esta región es atractiva para la instalación de plantas solares (Narvaez, et al, 2008).

En España, realizo una investigación sobre medida y modelización de la irradiancia solar eritemática global sobre planos inclinados, Respecto al MAD y RMSD relativos, los resultados son peores que cuando se consideran todas las condiciones de cielo en las orientaciones norte, este y oeste (Serrano, et al 2010 pp.57-66). Condiciones muy similares a las nuestras.

\section{OBJETIVOS}

a) Determinar y correlacionar los factores climáticos de acuerdo a las horas sol y radiación solar, que influyen para implementar positivamente proyectos de ERNC en las tres zonas de estudios (pampa Dos Cruces, cuesta El Águila y pampa Camarones), en relación a temperatura, humedad y velocidad del viento.

b) Medir la zona de pampa Dos Cruces, la que presente mayor área (ha), pendiente (\%) y altitud (msnm), en relación a las zonas de cuesta El Águila y pampa Camarones. 


\section{METODOLOGÍA}

Investigación inferencial, correlacional. Se analizaron 3 zonas correspondientes a la Depresión Intermedia del desierto seco, con una extensión que alcanza unos $40 \mathrm{~km}$ de ancho y cuyas alturas van desde 500 a 2900 msnm. Correspondiente a la Región de Arica y Parinacota, administrada territorialmente por 4 comunas de Arica, Putre, Camarones y General Lagos, de la Región XV de Chile con una superficie total de $16.873,30 \mathrm{~km}^{2}$ y una población de 226068 . La recopilación de datos considera el $100 \%$ de la muestra de estaciones meteorológicas, en el parámetro de factores climáticos como temperatura, humedad y velocidad del viento, para los meses de estudio. Se aplica análisis de varianza (ANDEVA), midiendo la variación de las zonas de estudio de pampa Dos Cruces, la que presente mayor área (ha), pendiente (\%) y altitud (msnm), en relación a las zonas de cuesta El Águila y pampa Camarones. El contraste del grado de asociación entre Temperatura ${ }^{\circ} \mathrm{C}$ y Humedad ${ }^{\circ} \mathrm{H}$, es analizado a través de la aplicación de la prueba estadística de correlación con un nivel de confianza del $95 \%$ alfa $=0.05$ utilizando el programa de Infoestat versión libre. Se instalará una estación radiométrica en el municipio de Arica (Chile), la estación estará sujeta a 4 comprobaciones:

1. Revisión Visual, hecha por un operador entrenado, preestablecerá la posible existencia de incidencias en cada uno de los días.

2. Comprobaciones de Primer Nivel, aquellas destinadas a evaluar si cada una de las medidas obtenidas, están dentro de los límites permitidos.

3. Comprobaciones de Segundo Nivel, aquellas destinadas a evaluar si cada una de las medidas obtenidas están dentro de los límites extremadamente raros.

4. Comprobaciones de Tercer Nivel, aquellas destinadas a evaluar si las medidas de irradiación solar obtenidas, son consistentes entre sí.

Tabla 1

Instrumentos de Medida y Detalle de Medición.

\begin{tabular}{cc}
\hline Instrumento de Medida & Detalle de Medición \\
\hline Piranómetro & Radiación global, directa, difusa reflejada \\
Pirheliómetro & Radiación solar, directa e incidencia normal \\
Actinógrafo & Radiación global \\
Heliógrafo & Brillo solar \\
\hline
\end{tabular}

\section{RESULTADOS}

En el mismo esquema de las horas de sol y los meses del año, en la Región de Arica y Parinacota los resultados obtenidos muestran que, en los meses de enero hasta abril, comienza a descender las horas de sol hasta julio, luego a partir de agosto nuevamente se incrementa (Fcalc (0.05) = 572.42; gl=8; $P=0.0001$ ) (Fig. 1)

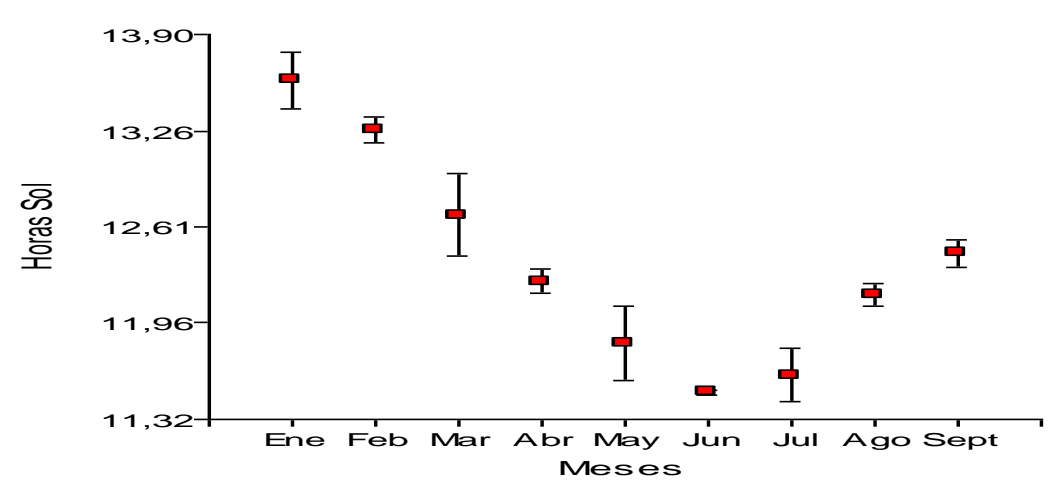

Figura 1 Medición De Las horas de sol y meses de año entre los periodos de enero a septiembre 2018. 
Respecto de las horas Sol e Irradiancia en el sector de Pampa Dos Cruces, los resultados obtenidos presentan una asociación de $r=0,43$. Respecto de las horas de Sol e Irradiancia en el sector quebrada El Águila, los resultados obtenidos presentan una asociación de $r=0,70$ (Tabla 2)

Tabla 2 Horas Sol / Irradiancia en Sector Pampa Dos Cruces y en Sector Quebrada El Águila.

\begin{tabular}{llr}
\hline Correlación de Pearson: Coeficientes Iprobabilidades & Horas Sol & Irradiancia Dos Cruces \\
\hline Horas Sol & 1,00 & 0,00 \\
Irradiancia Dos Cruces & 0,43 & 1,00 \\
\hline Correlación de Pearson: Coeficientes Iprobabilidades & Horas Sol & Irradiancia El Águila \\
\hline Horas Sol & 1,00 & 0,00 \\
Irradiancia El Águila & 0,70 & 1,00
\end{tabular}

Fuentes. Dirección de Aeronáutica Civil AP Chacalluta / Estaciones meteorológicas Engie.cl 2018

Respecto a las horas Sol y Temperatura en el sector de pampa Dos Cruces, EL Aguila y Pampa de camarones, los resultados obtenidos presentan una asociación de $r=0,8$. (Tabla 2)

Tabla 3 Horas Sol / Temperatura en Sector Pampa Dos Cruces, en Sector Quebrada El Águila y en Sector Pampa Camarones

\begin{tabular}{llc}
\hline Correlación de Pearson: Coeficientes|probabilidades & Horas Sol & Temperatura Dos Cruces \\
\hline Horas Sol & 1,00 & 0,00 \\
Temperatura Dos Cruces & 0,80 & 1,00 \\
\hline Correlación de Pearson: Coeficientes Iprobabilidades & Horas Sol & Temperatura El Águila \\
\hline Horas Sol & 1,00 & 0,00 \\
Temperatura El Águila & 0,82 & 1,00 \\
\hline Correlación de Pearson: Coeficientes lprobabilidades & Horas Sol & Temperatura Camarones \\
Horas Sol & 1,00 & 0,00 \\
Temperatura Camarones & 0,81 & 1,00 \\
\hline
\end{tabular}

Fuentes. Dirección de Aeronáutica Civil AP Chacalluta / Estaciones meteorológicas Engie.cl, 2018

Respecto a las horas Sol y Humedad en los sectore estudiados, los resultados obtenidos presentan una asociación de $r=0,58$. (Tabla 4)

Tabla 4 Horas Sol / Humedad en Sector Pampa Dos Cruces, en sector Quebrada El Águila y en Sector Pampa Camarones

\begin{tabular}{lcc} 
Correlación de Pearson: Coeficientes \probabilidades & Horas Sol & Humedad Dos Cruces \\
\hline Horas Sol & 1,00 & 0,00 \\
Humedad Dos Cruces & 0,58 & 1,00 \\
\hline Correlación de Pearson: Coeficientes \probabilidades & Horas Sol & Humedad El Águila \\
\hline Horas Sol & 1,00 & 0,00 \\
Humedad El Águila & 0,58 & 1,00 \\
\hline Correlación de Pearson: Coeficientes\probabilidades & Horas Sol & Humedad Camarones \\
\hline Horas Sol & 1,00 & 0,00 \\
Humedad Camarones & 0,51 & 1,00 \\
\hline
\end{tabular}

Fuentes: Dirección de Aeronáutica Civil AP Chacalluta / Estaciones meteorológicas Engie.cl, 2018

Respecto a las horas Sol y la Velocidad del Viento en el sector de Pampa Dos Cruces, los resultados obtenidos presentan una asociación de $r=0,54$. En la zona de EL Águila fue de 0,34 y en Pampa de Camarones fue de 0,24. (Tablas 5) 
Tabla 5 Horas Sol / Velocidad Del Viento en Sector Pampa Dos Cruces, en Sector Quebrada El Águila y en Sector Pampa Camarones

\begin{tabular}{|c|c|c|}
\hline \multicolumn{3}{|c|}{ Correlación de Pearson: Coeficientes\probabilidades } \\
\hline \multicolumn{3}{|r|}{ Velocidad Viento Dos Cruce } \\
\hline Horas Sol & 1,00 & 0,00 \\
\hline Velocidad Viento Dos Cruce.. & 0,54 & $1,0 \underline{0}$ \\
\hline \multicolumn{3}{|c|}{ Correlación de Pearson: Coeficientes\probabilidades } \\
\hline \multicolumn{3}{|c|}{ Horas Sol Velocidad Viento El Águila.. } \\
\hline Horas Sol & 1,00 & $1,0 \mathrm{E}-08$ \\
\hline Velocidad Viento El Águila & 0,34 & 1,00 \\
\hline \multicolumn{3}{|c|}{ Correlación de Pearson: Coeficientes\probabilidades } \\
\hline & Horas Sol & Viento Camarones. \\
\hline Horas Sol & 1,00 & $6,2 \mathrm{E}-05$ \\
\hline Velocidad Viento Camarones & 0,24 & 1,00 \\
\hline
\end{tabular}

Las mayores pendientes (\%), la presenta la zona de Pampa dos Cruces con un promedio de $52,6 \%$ que sus menores como Pampa Camarones con un promedio de $31,3 \%$ y por último quebrada El Águila con 16,5\% (Fcalc (0.05) = 39.250; gl=20; P=0.0001) (Figura 2).

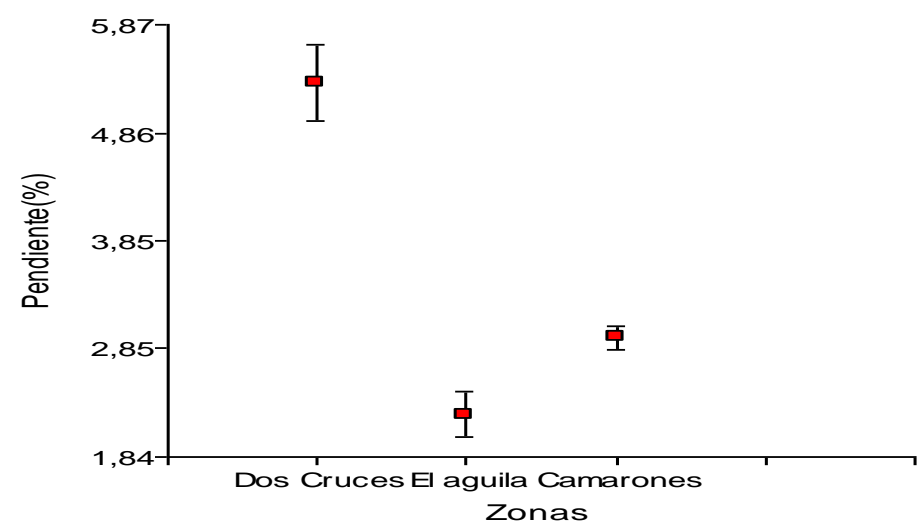

Figura 2 Variación De Pendiente (\%) Entre Las 3 Zonas De Estudio.

Las mayores altitudes (msnm), se presenta en la zona de quebrada El Águila con un promedio de 1649.36 msnm, mientras que en Pampa Camarones con un promedio de $999.9 \mathrm{msnm}$. y finalmente Pampa Dos Cruces con un promedio de $678.32 \mathrm{msnm}$. (Fcalc (0.05) = 57.730; gl=20; $\mathrm{P}=0.0001$ ) (Figura 3).

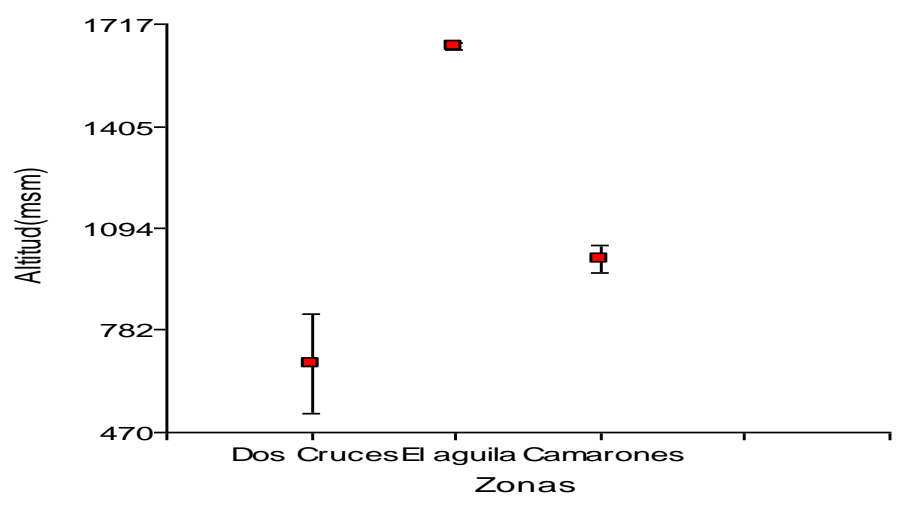

Figura 3 Variación De Altitud (Msnm) Entre Las 3 Zonas De Estudio. 


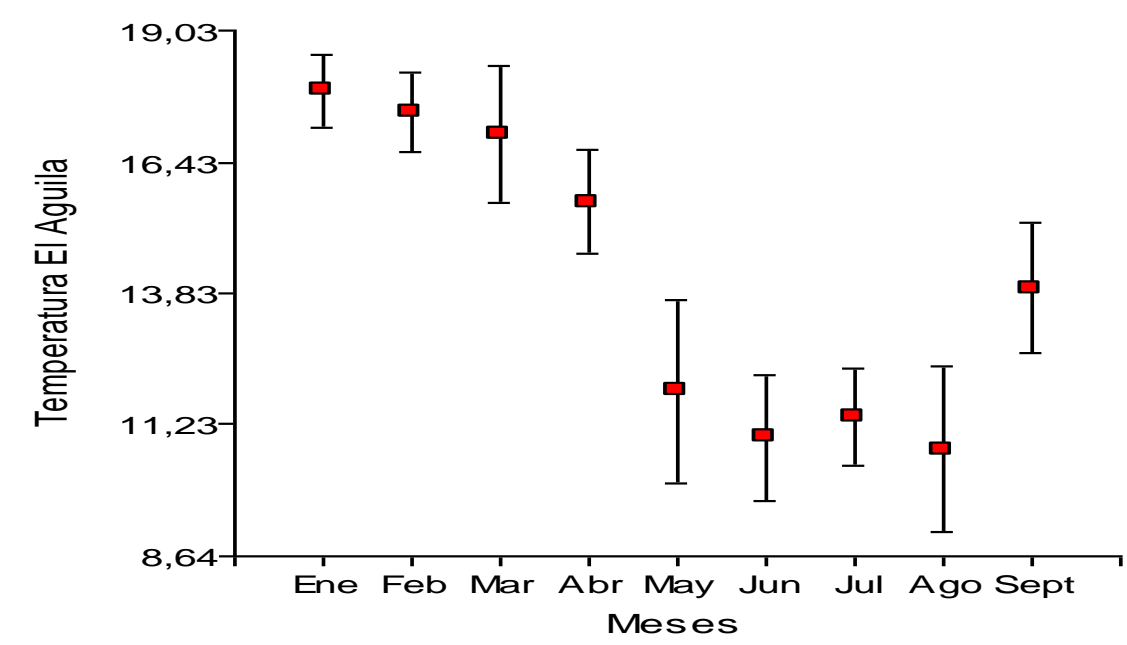

Figura 4 Variación de temperatura ambiental entre los meses de enero a septiembre 2018 en la zona del Águila Arica Chile

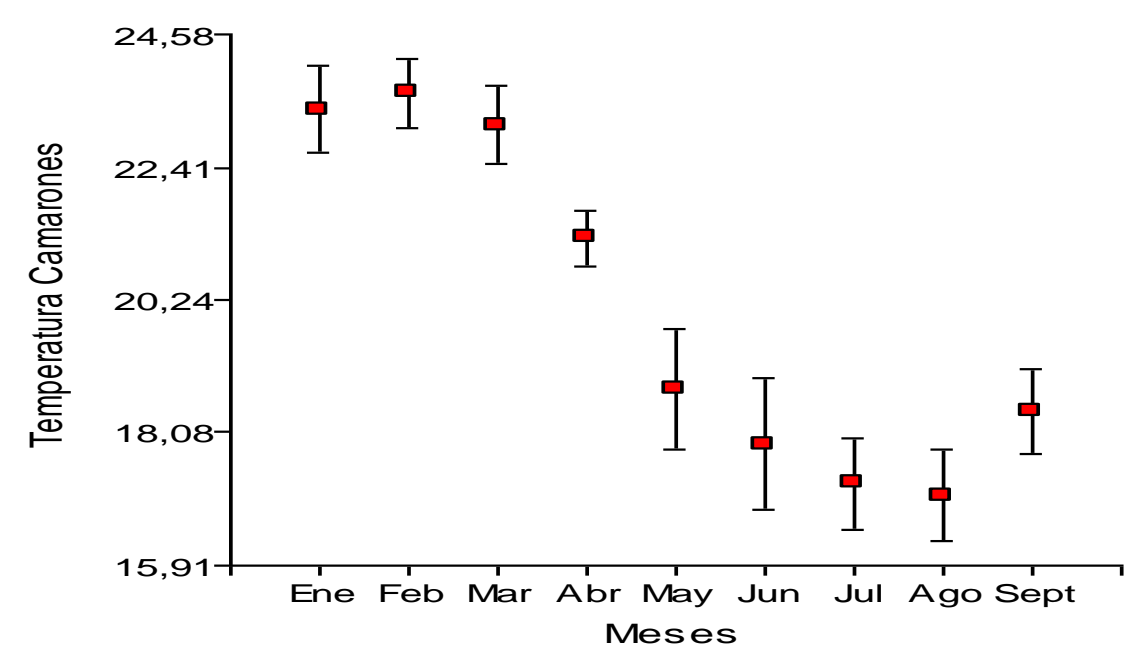

Figura 5 Variación de temperatura ambiental entre los meses de enero septiembre 2018 en la zona Pampa de Camarones Arica Chile.

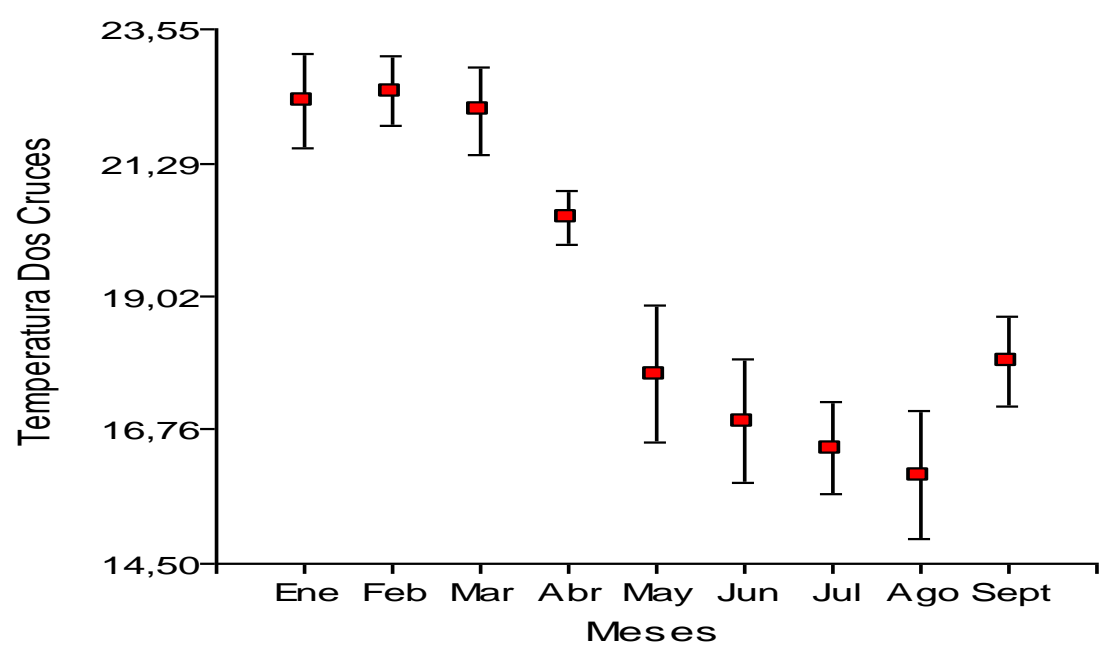

Figura 6 Variación de temperatura ambiental entre los meses de enero a septiembre 2018 en la zona pampa dos cruces Arica Chile 


\section{DISCUSIÓN}

Si comparamos los estudios del año 2002 con los recientes moniteoreos del año 2018, se puede indicar que se presenta una tendencia en relación a las horas de sol versus los meses calendarios, esto se ratifica según (Rivas et al 1995), (Rivas et al., 1996a; Rivas et al.,1996b; Rivas et al., 1997). estudio que ratifica que Arica, en la zona tropical del norte de Chile, presenta un microclima de características singulares, con condiciones meteorológicas notablemente estables durante todo el año. De acuerdo a las gráficas los monitoreos del año 2018, demuestran que los meses de primavera y verano tienen una mayor irradiación solar con escasa nubosidad, lo que acompañado a más horas luz, los niveles de radiación son mayores, esto lo ratifican los estudios de (Luccini et. al, 1998), los cuales indican que, dada la ubicación intertropical del lugar, el Sol pasa dos veces por el zenit durante el verano, con lo cual el período de altos niveles de radiación se extiende ampliamente. Registros del orden de (ISUV) $=12$ se dan durante los máximos de fin de primavera y principios de verano, de igual manera en la ciudad Arica se observan valores iguales o superiores a (ISUV) = 12 a mediodía solar durante la mayor parte de primavera y verano.

Entre los modelos desarrollados para estimar la radiación solar, se incluyen la ecuación de Ångström-Prescott y modificaciones de ésta, modelos lineales, polinómicos, exponenciales y logarítmicos (Menges et al., 2006); también estocásticos y de redes neuronales, entre otros (Mellit et al., 2005; Raichijk, 2008; Bocco et al., 2010; Kaplanis y Kaplani, 2010). En particular (Fadare et al. 2010) diseñaron diferentes redes neuronales del tipo multicapa con el algoritmo backpropagation para predecir radiación solar media mensual en África, utilizando como variables de entrada siete parámetros entre geográficos y meteorológicos; (Leal et al. 2009) elaboraron modelos estadísticos para estimar radiación solar ultravioleta diaria para dos localidades de Brasil, y (Spokas y Forcella 2006) desarrollaron modelos empíricos para estimar radiación solar total horaria para 18 sitios ubicados en USA y Canadá.

De acuerdo a los monitoreos obtenidos, se puede concluir que la relación de horas sol y temperatura ambiente, en la zona catastrada, esta tiene una tendencia promedio, donde las zonas de pampa Dos Cruces ( $r=0.80$ ), el promedio de $R$ está en la media de los 0.80 , el resultado de estos análisis lo ratifica el modelo utilizado por (Di Rienzo et al., 2010) que analizando los resultados con el software Infostat, se observa correlación positiva entre la radiación observada y las variables temperatura $(R=0,61)$ y velocidad del viento $(R=0,31)$ y correlación negativa para humedad $(R=-0,56)$, no existiendo prácticamente correlación con la precipitación $(R=-$ 0,05).

En referencia, a la zona monitoreada de Quebrada El Águila presenta una tendencia promedio $(r=0.82)$, donde se puede concluir que la relación de horas sol y temperatura ambiente, donde el promedio de $\mathrm{R}$ está en la media de los 0.80 , este resultado de estos análisis lo ratifica el modelo utilizado por (Di Rienzo et al., 2010) que analizando los resultados con el software Infostat, se observa correlación positiva entre la radiación observada y las variables temperatura.

Otro elemento comparativo para este estudio, son el uso directo de las variables que presentan únicamente las estaciones que, como la citada, son de uso frecuente en productores y estaciones experimentales. De hecho, (Mellit et al. 2005), mediante una red RBF estimaron radiación solar diaria en Argelia usando heliofanía, temperatura y radiación solar global con un coeficiente de determinación de 0,96. (Bocco et al. 2010) lo hicieron para Salta, a través de una red tipo perceptrón y obtuvieron un valor de $\mathrm{R} 2=0,92$.

En la zona monitoreada de pampa Camarones tiene una tendencia promedio $(r=0.81)$, donde el promedio de $\mathrm{R}$ está en la media de los 0.80 , la característica de esta zona de estudio la compone 
su cercanía al mar lo que influye notoriamente en su posicionamiento geográfico, respecto a las otras dos zonas de estudio, de acuerdo a lo indicado por (Fuenzalida, 1971) La circulación atmosférica superficial a gran escala de la región centro y norte de Chile, es dominada por la presencia del anticiclón subtropical del Pacífico Sur Oriental (APSO). SU centro se localiza entre los $25^{\circ}$ y $30^{\circ} \mathrm{S}$ y los $90^{\circ}$ y $105^{\circ} \mathrm{W}$ y su influencia típicamente alcanza los $38^{\circ} \mathrm{S}$, aun cuando durante el verano austral éste puede penetrar hacia el sur sobrepasando los $45^{\circ} \mathrm{S}$. De igual forma si comparamos nuestro monitoreo con los estudios realizados por (Vuille et al 2015), el cual analizo las zonas costeras, valles, quebradas y pre-altiplánicas, donde se puede constatar una tendencia en las temperaturas como lo indica la estación Codpa, la cual Estación ubicada a 114 kilómetros al Sureste de Arica, en el corazón de la quebrada de Camarones a $1870 \mathrm{msnm}$. Esta estación representa uno de los mejores ejemplos en cuanto al aumento de las temperaturas máximas tal como lo expone el IPCC 2013, donde la temperatura media global aumentó en $0,85^{\circ} \mathrm{C}$ en los últimos 30 años; y donde las mínimas muestran una disminución en promedio de $0,5^{\circ} \mathrm{C}$; acentuando aún más las condiciones de continentalidad y oscilación térmica.

De acuerdo a los resultados del monitoreo en las 3 zonas catastradas, se puede concluir que la relación entre pendiente (\%) y superficie $(\mathrm{Ha})$, depende de la morfología del relieve ya que existen rasgos muy distintos entre ellas, donde pampa Dos Cruces y pampa Camarones estos conformados desde un acantilado costero del orden de los $1000 \mathrm{msnm}$, en cambio quebrada El Águila, son las grandes quebradas formadas por profundos cañones de más de $600 \mathrm{~m}$, cuyas incisiones en sentido este-oeste, cortan transversalmente las unidades del relieve desde el límite occidental de la Cordillera de los Andes, esto asegurado por (Brüggen, 1950), debido a su gran altitud y pendiente, estableció que tenía un origen tectónico, correspondiendo a un escarpe de falla. Esta idea fue retomada por (Armijo \& Thiele, 1990) sobre la base de la actividad de fallas normales en la península de Mejillones y Zona de Falla de Atacama, por otro lado (Mortimer \& Saric,1972) no explican la causa y mecanismo de subsidencia litoral.

El efecto de la radiación solar tiene mucho que ver con la ubicación geográfica y la altitud en relación al nivel del mar, considerando la superficie $(\mathrm{Ha})$, en relación a la altitud, nos entregan datos bien marcados, esto queda demostrado en los cálculos mediante el modelo TUV se realizaron en un período comprendido entre los años 1996-2003, introduciendo los parámetros de los lugares geográficos en que se hicieron las mediciones experimentales, (Rivas et al 2008), de igual forma estas mediciones para calcular el efecto altitudinal han sido realizadas en el paso por (Cabrera, et al 1985), quien midió con un radiómetro PUV-510 en las bandas de 305, 320, 340 y $380 \mathrm{~nm}$, con un ancho de banda de $10 \mathrm{~nm}$. Las mediciones fueron realizadas durante el verano e invierno en Arica (Lat. 18 20'S; long. 70 19' W, h = $20 \mathrm{~m}$ ) y Putre (Lat.18ㅇ 20' S; long. 69 32' W, h = $3.460 \mathrm{~m}$ ), en las bandas de 305, 320, 340, $380 \mathrm{~nm}$; los porcentajes de aumento de irradianza UV por cada $1.000 \mathrm{~m}$ de altitud sobre el nivel del mar, fueron de un 5,1 \% en verano y un $5,8 \%$ en invierno por cada $1.000 \mathrm{~m}$ de altitud.

En relación a las condiciones climáticas de la zona, que para los datos obtenidos corresponden a un desierto marginal de altura, con escasas precipitaciones en época del invierno y temperaturas que muestran un ascenso en los valores medios para las últimas décadas, como lo demuestran los datos obtenidos, como por ejemplo del monitoreo de la planta de Pampa Camarones. En los meses de verano existe más horas luz y por ende mayor irradiancia solar a diferencia de los meses de invierno donde decae, tanto en luz día e irradiancia. Lo que nos indica positivamente las inmejorables condiciones que existen en el territorio tanto climáticas como geomorfológicas para la futura instalación de proyectos de energía renovable no convencionales, (ERNC), a través de la tecnología solar fotovoltaica. 
Se demostró que la pendiente y altitud comparativamente hacen la diferencia entre una zona y otra, los datos indican que las mayores pendientes (\%), la presenta la zona de Pampa dos Cruces con un promedio de $52,6 \%$ que sus menores como Pampa Camarones con un promedio de $31,3 \%$ y por último quebrada El Águila con $16,5 \%$ a diferencia del factos de la altitud (msnm), donde quebrada El Águila con 2727.93 msnm se encuentra por sobre los $1000.00 \mathrm{msnm}$ promedio que las otras dos zonas, lo que permite que las condiciones climáticas en esta zona sean las más optimas por su escasa nubosidad.

\section{REFERENCIAS BIBLIOGRÁFICAS}

Aguilar, G., Riquelme, R., Martinod, J., \& Darrozes, J. (2013). Rol Del Clima Y La Tectónica En La Evolución Geomorfológica de Los Andes Semiaridos Chilenos entre los 27-32º. Revista Geologica de Chile, V.40, pp. 79-101. doi:doi: 10.5027/andgeoV40n1-a04

Fabry Ch., B. (1913). Étude de l'extrémité ultra-violette du spectre solaire. J. Phys Radium. Obtenido de https://hal.archives-ouvertes.fr/jpa-00204283

Facultad de Ciencas Físcicas y Matematicas Universidad de Chile. (2012). http://walker.dgf.uchile.cl/Explorador/Solar2/info/Documentacion_Explorador_Solar.pdf. Obtenido http://walker.dgf.uchile.cl/Explorador/Solar2/info/Documentacion_Explorador_Solar.pdf

Falvey, M., \& Garreaud, R. (2009). Enfriamiento regional en un mundo en calentamiento. Department of Geophysics, Universidadde Chile, V. 114, p. 45. doi:doi:10.1029/2008JD010519

IPCC Grupo Intergubernamental de Expertos sobre Cambios Clímaticos. (1997). Libro de Trabajo para EI Inventario de Gases de Efecto Invernadero. Reino Unido.

IPCC Grupo Intergubernamental de Expertos sobre el Cambio Climático. (2013). Cambio Clímatico 2013 Resumen para Resposables de Políticas. Suiza. Obtenido de www.climatechange2013.org

IPCC, C. G. (2002). Cambio Climático y Biodiversidad. Paris. Obtenido de https://archive.ipcc.ch/pdf/technical-papers/climate-changes-biodiversity-sp.pdf

Lapa Camayo, F., Hurtado Condezo, E., Cadillo Ramos, Y., Cadillo Ramos, R., \& Vivas camayo, B. (2019). Estimación de la radiación solar global, mediante temperaturas extremas, aplicando el modelo Bristow-Campbell en la región Junín, Perú. Revista Chilena de Ingeniería, V.27(N4), pp.220-234. Obtenido de http://dx.doi.org/10.4067/S0718-33052019000400643

MINISTERIO DE SALUD. (2012). ANÁLISIS DE SITUACIÓN. Lima-Perú: ASKHA E.I.R.L.

Narvaez, J., Dielman, G., \& Gordillo, G. (2008). Estudio del Potencial de Radiación Solar y de la Temperatura Ambiente en Lorica-Cordoba. Rev. Colombiana de Física, V.4O(N²).

Rivas A., M., Rojas E., E., Cortés E., J., \& Santander G., E. (2002). Efecto de la Altura en la Radiación Solar Ultravioleta en Arica Norte de Chile. Facultad de Ingeniería UTA, Chile, 10, pp. 59 -62.

Rivas A., M., Rojas E., E., Cortés N., J., \& Santander G., E. (2002). Datos Experimentales de Indice de Riesgo Solar Medidos en Arica, Norte de Chile durante el año 2002. Facultad de Ingeniería Universidad de Tarapaca, 10, p. 61.

Sarricolea Espinoza, P., \& Romero Aravena, H. (2015). Variabilidad y cambios climáticos observados y esperados en el Altiplano del norte de Chile. Revista de Geografía Norte Grande( $\left.{ }^{\circ} 62\right)$. Obtenido de http://dx.doi.org/10.4067/S0718-34022015000300010

Sarricolea, P., Meseguer ruiz, O., \& Romero Aravena, H. (2017). Tendencias De La Precipitación En El Norte Grande de Chile y su Relación con Las Proyecciones de Cambio Climático. Dialogo Andino( $\left.\mathrm{N}^{\circ} 54\right)$, pp. 41-50.

Sayago, S., Bocco, M., Ovando, G., \& Willington, E. (2011). Radiación solar horaria: modelos de estimación. Avance en energias Renovables y Medio Ambiente, 15.

Serrano, D., Marín, M., Ultrillas, M., Tena, F., \& Martínez Lozano, J. (2010). Medida y Modelización de la Irradiancia Solar Eritematica Gobla sibre Planos Inclinados. ACAM, pp. 57-66. doi:103369/tethys 5.7.2010. S

Velez P., A., Vergara V., E., Barraza C., W., \& Agudelo Y., D. (2015). Evaluación De Un Modelo Estadístico Para Estimar La Radiación Solar en Magdalena, colombia. Rev. Tecno Lógicas, V.18(N³5), pp.3544. 